\title{
Radiated Sound Power from a Curved Honeycomb Panel
}

\author{
Jay H. Robinson* \\ Ralph D. Buehrle ${ }^{\dagger}$ \\ Jacob Klos ${ }^{\dagger}$ \\ NASA Langley Research Center, Hampton, VA 23681-2199 \\ Ferdinand W. Grosveld ${ }^{\ddagger}$ \\ Lockheed Martin Engineering and Sciences, Hampton, VA 23681
}

\begin{abstract}
The validation of finite element and boundary element model for the vibro-acoustic response of a curved honeycomb core composite aircraft panel is completed. The finite element and boundary element models were previously validated separately. This validation process was hampered significantly by the method in which the panel was installed in the test facility. The fixture used was made primarily of fiberboard and the panel was held in a groove in the fiberboard by a compression fitting made of plastic tubing. The validated model is intended to be used to evaluate noise reduction concepts from both an experimental and analytic basis simultaneously. An initial parametric study of the influence of core thickness on the radiated sound power from this panel, using this numerical model was subsequently conducted. This study was significantly influenced by the presence of strong boundary condition effects but indicated that the radiated sound power from this panel was insensitive to core thickness primarily due to the offsetting effects of added mass and added stiffness in the frequency range investigated.
\end{abstract}

\section{Nomenclature}

F complex force

$V \quad$ complex velocity

$c \quad$ damping parameter

$i \quad \sqrt{-1}$

$k \quad$ stiffness parameter

$\Omega \quad$ natural frequency, $\mathrm{rad} / \mathrm{sec}$

$\omega \quad$ frequency, $\mathrm{rad} / \mathrm{sec}$

\section{Superscripts \\ $i \quad$ index of summation \\ $n, t \quad$ edge normal and tangential components \\ $r \quad$ surface normal or radial component}

\section{Introduction}

One goal of NASA's airframe noise reduction program is to identify, develop, and demonstrate technologies to achieve a 4 decibel reduction in interior noise with minimal or no weight penalties for advanced structural concepts. One such concept of interest uses honeycomb composite panels as the primary load carrying structure in the aircraft fuselage. To investigate the acoustic characteristics of curved honeycomb

\footnotetext{
*Aerospace Engineer, Multidisciplinary Optimization Branch, Aerospace Systems, Concepts \& Anal Comp.

${ }^{\dagger}$ Aerospace Engineer, Structural Acoustics Branch, Aerodyns, Aerothermodyns \& Acoust Comp.

¥Aerospace Engineering Manager, Lockheed Martin Corp. Associate Fellow AIAA

This paper is a work of the U.S. Government and is not subject to copyright protection in the United States.
}

composite panels, a representative panel was fabricated and tested in the Structural Acoustics Loads and Transmission (SALT) Facility ${ }^{1}$ at NASA Langley Research Center. A schematic of the SALT facility is shown in figure 1. Experiments by Klos et. al. ${ }^{2}$ showed that these honeycomb composite panels exhibit poor acoustic characteristics primarily because of their high stiffness to weight ratio. Initial investigations, reported by Buehrle et. al. ${ }^{3}$ and Grosveld et. al., ${ }^{4}$ focused on verifying the structural acoustic modeling techniques required for this type of panel. In these studies the authors demonstrated the validity of the finite element model of the curved honeycomb panel with free-free boundary conditions by a comparison of measured and predicted natural frequencies. The authors also validated the boundary element model by projecting a measured velocity field onto the boundary element mesh and accurately predicting the radiated acoustic pressure. The authors did not successfully validate the acoustic pressure prediction based on a finite element predicted velocity field. This was attributed to two problems. The first was in interpolating the finite element predicted velocity field onto the boundary element mesh. This was corrected in the current study by improving the interpolation method. The second and more difficult problem encountered was that of modeling the boundary conditions of the panel as it was installed in the test facility. As discussed in the previous investigations Buehrle et. al. ${ }^{3}$ and Grosveld et. al., ${ }^{4}$ the panel 


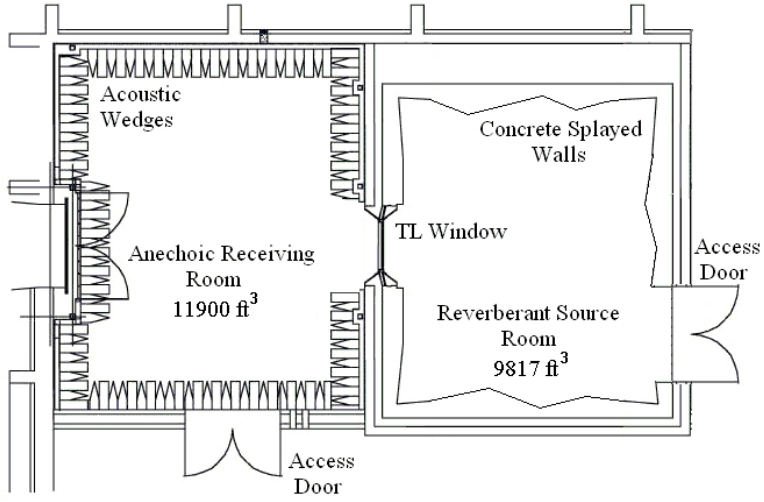

Fig. 1 Schematic of the Structural Acoustic Loads and Transmission (SALT) facility consisting of an anechoic chamber, a reverberation chamber, and a share transmission loss (TL) window.

was held in a fiberboard frame by means of a plastic gasket wedged between the panel and the frame. The plastic tubing was intended to eliminate acoustic flanking between the reverberant source room and anechoic receiving room of the SALT facility and to obtain spatially uniform boundary conditions. A cross section of the fiberboard fixture and plastic tubing is shown in Figure 2. The fixture is shown without the panel installed. This boundary condition has been extremely difficult to model due to it's frequency dependence and despite its intent spatially varying properties. The fiberboard frame with the panel installed was then mounted in the SALT transmission loss window as shown in Figure 3. The first section of the paper describes the steps taken beyond these two initial publications to improve the validity and accuracy of the finite element model.

In the second part of this paper, the new validated finite element model is used to investigate the influence of core thickness on the sound radiation characteristics of this curved honeycomb composite panel. This is the first of a number of investigations, both experimental and numerical, being conducted to ascertain how to improve the acoustic performance for this type of fuselage construction. Other investigations ${ }^{2}$ have been focusing on how to drive the bending and shear waves on the structure subsonic to reduce the radiation efficiency. This investigation focuses on the bending stiffness of the panel and its influence on radiated sound. Results will be presented in terms of a parametric study with the independent variable being the core thickness.

\section{Model Development}

For the purposes of this study, a curved sidewall panel was constructed from a flexible nomex honeycomb core sandwiched between carbon fiber reinforced composite laminate face sheets. The 2.06-centimeter $(\mathrm{cm})$ thick panel has a length of 1.39 -meter $(\mathrm{m})$, an

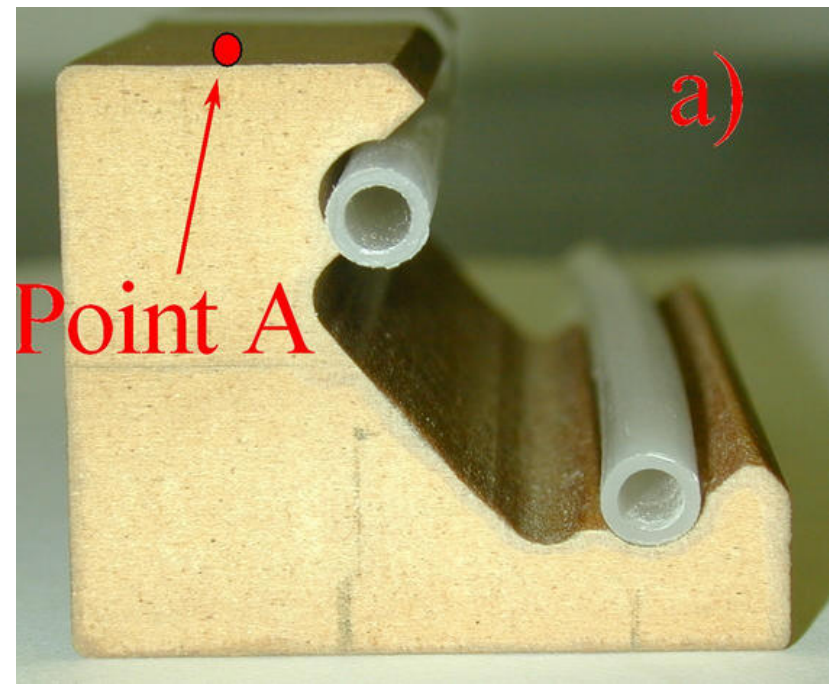

Fig. 2 Cross section of the fixture used to hold the panel in the transmission loss window without the composite panel installed.

inner radius of $1.04 \mathrm{~m}$, and covers an arc of approximately 82 degrees. A COMET/Acoustics boundary element model of the curved composite panel was developed and validated by Grosveld et. al. ${ }^{4}$ and Buehrle et. al. ${ }^{3}$ In this model the radiating surface of the composite panel was modeled using 24 QUAD4 elements in both the axial and arc directions. A total of 576 boundary elements were used to represent the surface velocity of the panel. This resolution resulted in adequate convergence of the radiated sound power up to $1000 \mathrm{~Hz}$ while still yielding reasonable computation times. The frame of the transmission loss window, shown in Figure 3, and the fiberboard fixture were also included in the boundary element model. To simulate the same acoustic boundary conditions present in the experiment, the elements used to model the frame and the fixture had a zero velocity boundary condition imposed. The authors validated the boundary element model by applying a measured velocity field onto the boundary element mesh and predicting the radiated acoustic pressure. These results are included in this investigation to help understand the validity of the acoustic pressure predictions based on the finite element predicted velocity field. This boundary element model is also used in this investigation to predict the sound power radiated from the panel as a result of a velocity response predicted using the finite element model.

The NASTRAN finite element model of the curved composite panel used in this investigation was also developed and validated by Grosveld et. al. ${ }^{4}$ for freefree boundary condition. The honeycomb core of the composite panel was modeled with NASTRAN solid CHEX-8 elements using the bulk properties of the honeycomb material. The face sheets were modeled with NASTRAN plate CQUAD-4 elements that were attached to the elements of the core at each grid point. 


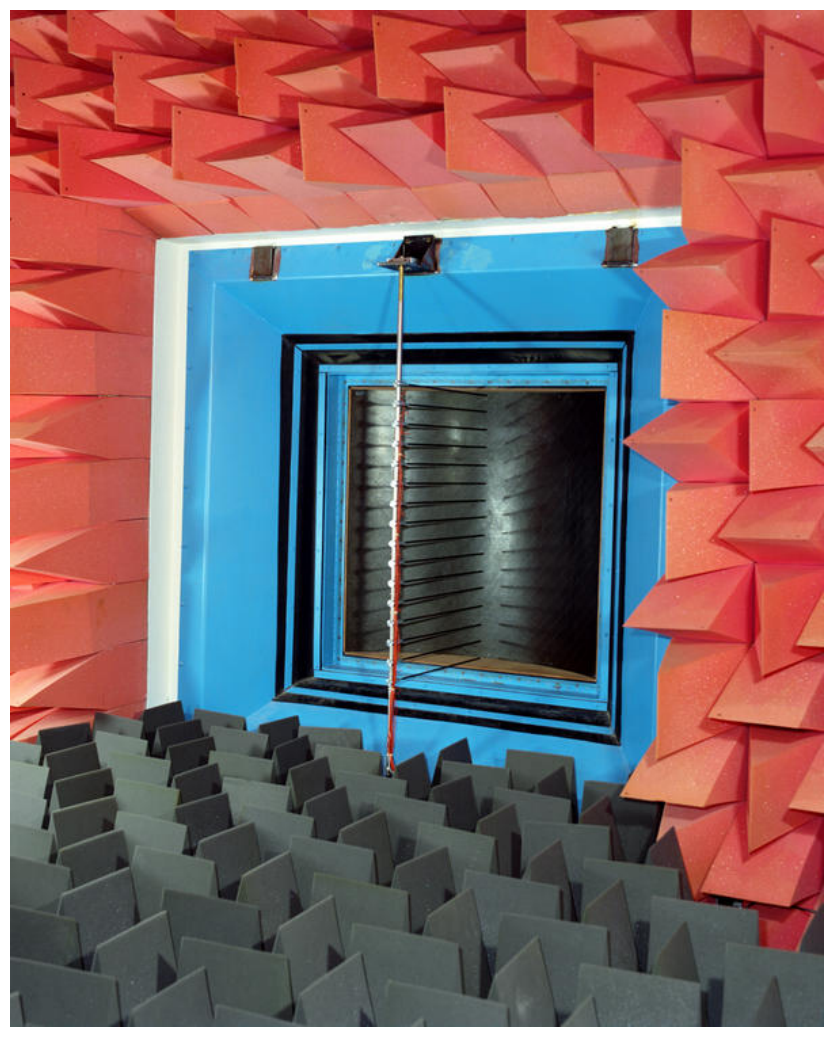

Fig. 3 Installation of the honeycomb core composite panel and experimental set up as viewed from the anechoic chamber of SALT.

Full allowance was made for anisotropy of the core material and orthotropy of the face sheets. There were 43 element in the arc direction, 43 elements in the axial direction, and the core was modeled with a single solid element through the thickness. Each face sheet was modeled using a single plate element through the thickness with the equivalent laminate properties. The element size ensured adequate convergence of the velocity response up to a frequency of $1000 \mathrm{~Hz}$. The initial validation of this model for free-free boundary conditions is discussed in a previous publication by Grosveld et. al. ${ }^{4}$ They reported that excellent agreement was obtained between predicted and measured natural frequencies for a large number of mode shapes. A subsequent comparison of the measure and predicted natural frequencies of the panel as installed the SALT facility proved less satisfactory when classical simply supported boundary conditions were applied to the finite element model. The authors attempted to improve their prediction by applying normal incident ground springs to the edges of their model. These were included in the NASTRAN model using CELAS1 elements. The magnitude of the spring stiffness was estimated by a parametric study and all springs were assumed to have the same stiffness. Though better agreement was obtained between the measured and predicted velocity responses the authors did not feel they successfully validated the acoustic pressure based on the finite element predicted velocity field. The conclusion was that the acoustic pressure predictions were significantly more sensitive to variations in velocity field induced by the boundary conditions than were evident by examination of the velocity responses themselves.

\section{Updated Model Development}

The primary deficiency noted in the previous studies was the model of the structural boundary conditions. At the end of these two previous studies the boundary conditions were modeled by ground springs normal to the surface of the panel. It is well established that mode shapes and response characteristics of curved cylindrical structures are influenced strongly by the inplane boundary conditions, see for example Leissa. ${ }^{5}$ It was also noted that the radiated pressure was even more sensitive to the in and out of plane boundary conditions than was the structural vibration response. For these reasons the model was updated using three ground springs on each edge of both the inner and outer face sheets of the honeycomb core composite panel. The first spring was the normal facing spring added in the previous investigation and the other two springs modeled the inplane stiffnesses normal and tangential to the free edges of the panel. The spring stiffnesses and their associated damping values were initially assumed to be independent of frequency and spatially uniform as before. These assumptions yielded six independent values. To determine the stiffness of each of the springs, an optimization procedure was used. The objective was to minimize the difference between measured and predicted frequencies for selected modes. The objective function was expressed as

$$
\operatorname{Obj}\left(k_{t}, k_{n}, k_{r}\right)=\sum_{i=1,6}\left(1-\frac{\omega_{i}}{\Omega_{i}}\right)^{2}
$$

where the measured frequencies are denoted as $\Omega_{i}$ and the predicted modal frequencies are denoted as $\omega_{i}$. The stiffness quantities $k_{t}, k_{n}$, and $k_{r}$ are the independent variables representing the inplane normal, inplane tangential, and surface normal spring stiffnesses respectively. The frequencies selected from the measured results for inclusion in the optimization procedure consisted of: one unsymmetric and one symmetric mode in both circumferential and axial direction; one zero order mode in the circumferential direction; and one higher order mode also in the circumferential direction. Equal values of damping were assumed for each CELAS1 element and a best guess value was used. These improvements were added to the NASTRAN model using CELAS1 elements.

Simultaneously with this optimization study, an experimental effort to measure the boundary conditions in the radial direction was also conducted. In this experiment an estimate of the boundary impedance 


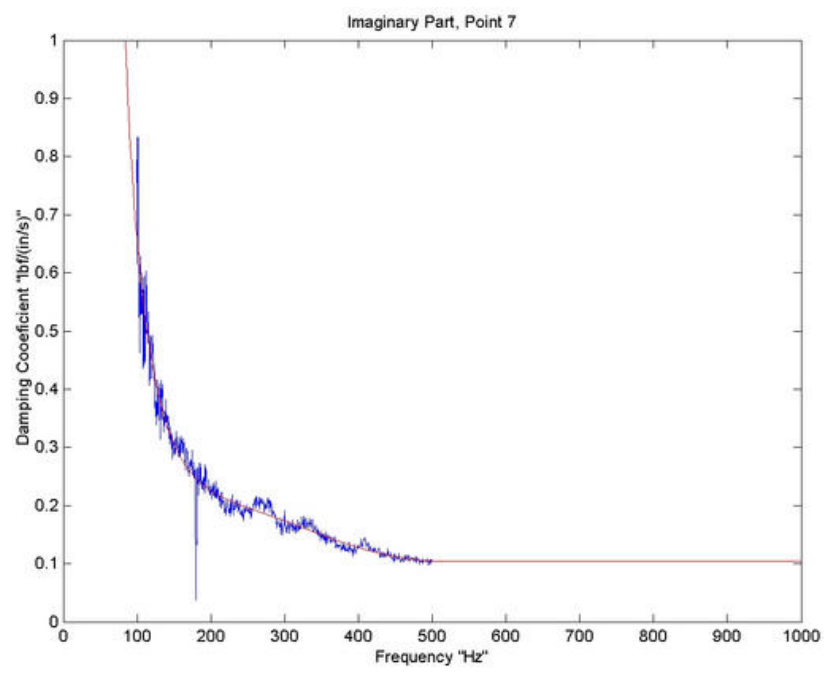

a) dissipation force component

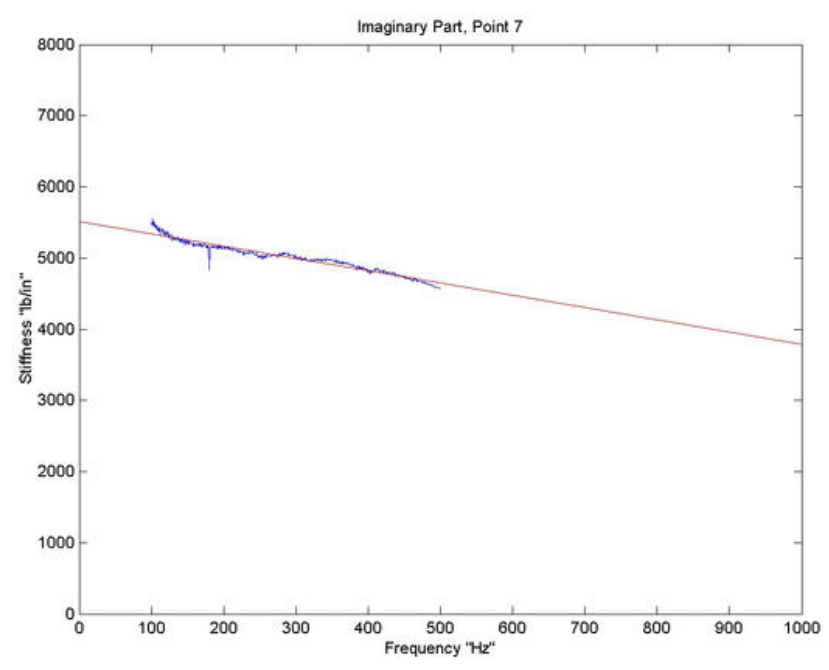

b) restoration force component

Fig. 4 Estimates of the frequency dependence of the damping and restoration forces extracted from an impedence measurement experiment.

was obtained by placing an impedance head on the panel adjacent to the boundary and driving it with a shaker. Measurements were made at three points on the panel approximately 1 inch from the point of contact between the plastic tubing and the composite panel. The first point was a quarter of the way up the straight edge of the panel, the second point was in the corner of the panel, and the third point was a quarter of the way around the circumference of the panel. The boundary stiffness and viscous damping coefficient were estimated from the measured impedance close to the boundary using

$$
\frac{F}{V}=\frac{k_{r}}{(i \omega)}+c
$$

where $\frac{F}{V}$ is the ratio of the complex force and complex velocity at the drive point. Thus, the boundary

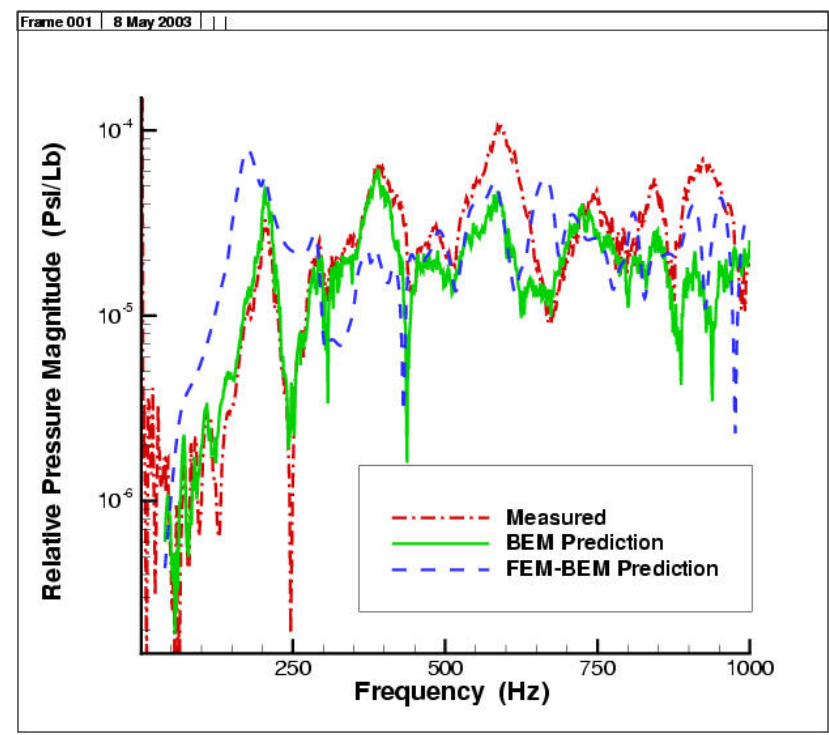

Fig. 5 Comparison of center far field microphone pressure versus frequency obtained from measurement, boundary element prediction using measured velocities, and combined finite element/boundary element prediction using measured structural excitation, respectively.

stiffness estimate is

$$
k_{r}=\omega \operatorname{Imag}\left(\frac{F}{V}\right)
$$

and the viscous damping coefficient estimate is

$$
c=\operatorname{Real}\left(\frac{F}{V}\right)
$$

There was good agreement between the measured stiffness at all three points and good agreement in the viscous damping coefficient found from the impedance measurements at two of the points. However, the viscous damping coefficient measured at the first point, a quarter of the way up the straight edge of the panel, was significantly higher than it was at the other points. This may be due to resonant behavior of the panel or fiberboard fixture corrupting the measurement. For this reason, the measurement at the point in the corner of the panel was used to extract the boundary stiffness and damping properties. The measured stiffness and damping coefficients at this point were fit with a linear and exponential curves respectively. The results of this curve fit are shown in Figure 4. The data from the curve fit were incorporated into the FE model of the composite panel using tabulated entries on the material property cards. The results of these two independent investigations were combined by using the frequency dependency obtained from the impedance measurements and the nominal values of stiffness obtained from the optimization study. The new model consisted of frequency dependent boundaries and the previously validated panel model. 


\section{Updated Model Results}

The vibration and acoustic response measurements were acquired as described in detail by Buehrle et. al. ${ }^{3}$ for a point force excitation from a shaker. The location of the shaker was in the lower left quadrant of the panel viewed from the anechoic chamber(Figure 3 ). This excitation location was used for all of the vibroacoustic validation tests presented in this paper and in the referenced papers ${ }^{3} .{ }^{4}$ A vertical array of sixteen equally spaced microphones is also shown in Figure 3. The array was mounted on a rotational mechanism that allowed for automated positioning of the microphones over an arc of eighty degrees. For this series of tests, the microphones were placed at a distance of $9.8 \mathrm{~cm}$ from the panel surface and used to measure the near field sound pressure over eighty degrees in five degree increments. Far field sound pressure measurements were acquired at four locations. The first far field microphone was set at the height of the panel center and at a distance of 3.7 meters from the center of curvature for the panel. The three remaining microphones were set at a distance of 1.2 meters in the vertical and horizontal directions from the center microphone. A random shaker input was used to excite the panel over a $1000 \mathrm{~Hz}$ frequency range. For each angular position of the near field microphone array, data was acquired for 100 averages, using a Hanning window, with 1000 $\mathrm{Hz}$ frequency range and a resolution of $1.25 \mathrm{~Hz}$. Spectra, coherence and frequency response function data were measured with the force input as the reference. In addition to the vibroacoustic measurements, the panel and shaker setup was used to measure the surface velocity data required as input to determine the velocity boundary conditions needed for the boundary element analysis.

Figures 5 and 6 show the results obtained using the updated model with respect to the predicted far field pressure obtained from both the experiment and the previous investigation using measured velocities. These figures show reasonable correlation in frequency up to about six hundred Hertz but with substantial differences in amplitude at various frequencies. Of particular interest in these plots are the presence of resonant peaks in the finite element/boundary element prediction that are not present in either of the other curves. One of the most dominant of these occurrs at a frequency just below 200 Hertz. The observation at this frequency and several others indicate the significance of the dynamic characteristics of the support structure and its numerical model on the radiated pressure response. Figures 7 and 8 show comparisons of the near field pressure distribution obtained by the three methods for frequencies of 202.5 Hertz and 588.5 Hertz respectively. Figure 7(c) in comparison to figures 7(a) and 7(b) also shows the strong influence of the boundary conditions on the spatial distribution of the near field pressure. The higher frequency near field pressure

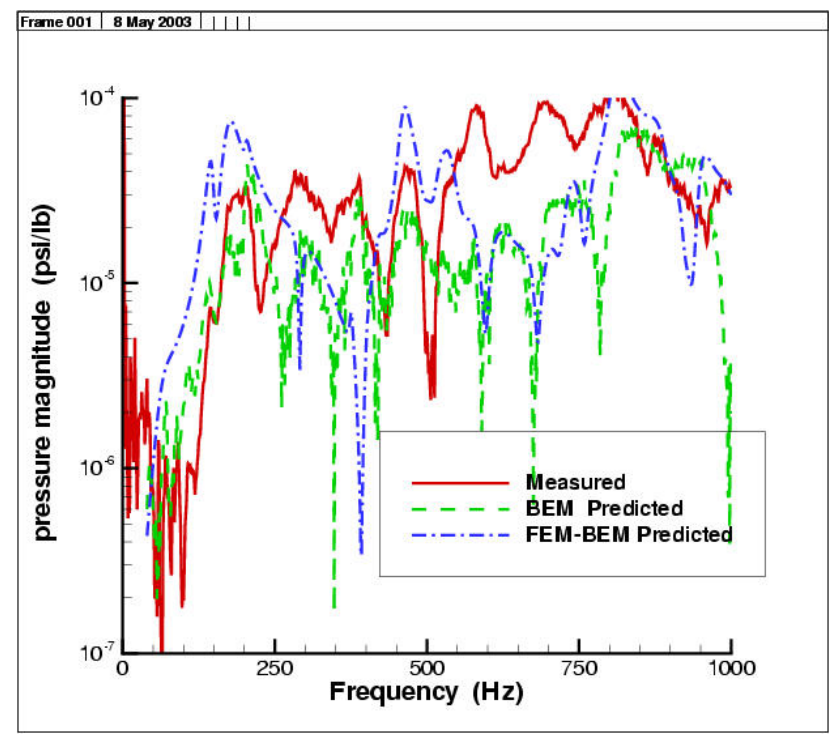

Fig. 6 Comparison of left of center far field microphone pressure versus frequency obtained from measurement, boundary element prediction using measured velocities, and combined finite element/boundary element prediction using measured structural excitation, respectively.

comparison shown in figures 8 is far more satisfactory and typical even though the magnitudes of the responses vary significantly. Though the correlation of the numerical model with respect to the measured responses is far from acceptable, the model is however believed to have sufficiently captured the physics of the experimental configuration up to about seven hundred Hertz. This is slightly short of the stated goal of one thousand Hertz.

\section{Parametric Study Results}

A parametric study was conducted to assess the sensitivity of the radiated sound from this panel with respect to a spatially uniform variation of the panel honeycomb core thickness. To insure that all vibration modes of the panel were equally excited an excitation representing a reverberant field was used in place of the point force excitation used for model validation. This field was created by summing a series of plane waves with random phases and angles of incidence to the panel's surface. The radiated sound power was calculated by integrating the normal intensity, as calculated by the boundary element program, over the surface of the panel and over the frequency range of the validated response. This frequencies ranged was taken to be from $40 \mathrm{~Hz}$ to $1024 \mathrm{~Hz}$ and a resolution of $1.25 \mathrm{~Hz}$ was used in the analysis. The variation of the core thickness ranged from 0.125 inches to 2.15 inches in increments of 0.1 inches. To further help characterize the panel behavior the parametric study was conducted for two different panels. The first was designated as the baseline panel with materials properties as specified by the manufacturer. The second panel 


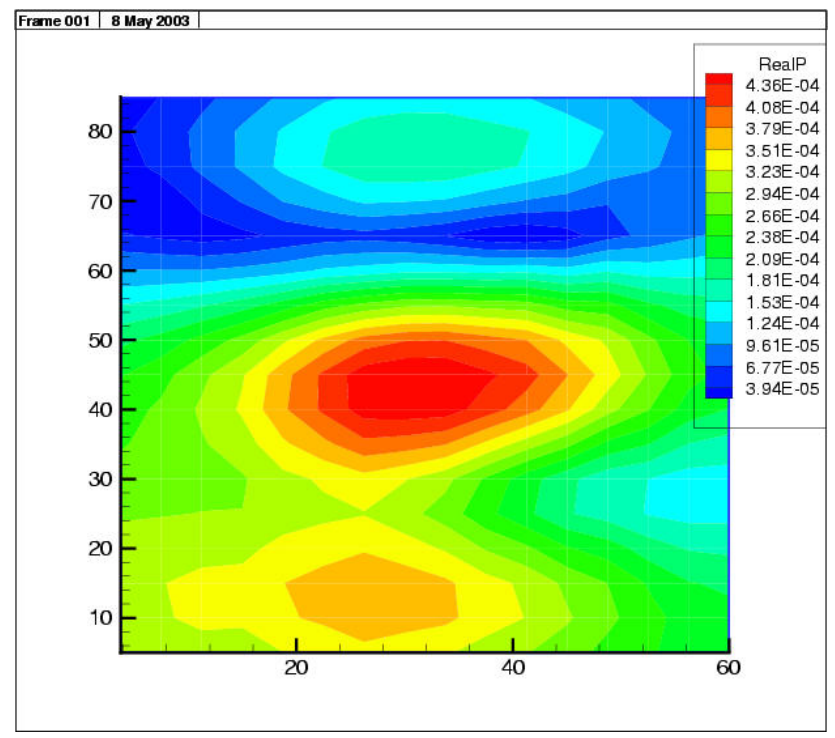

a) Magnitude of measured pressure.

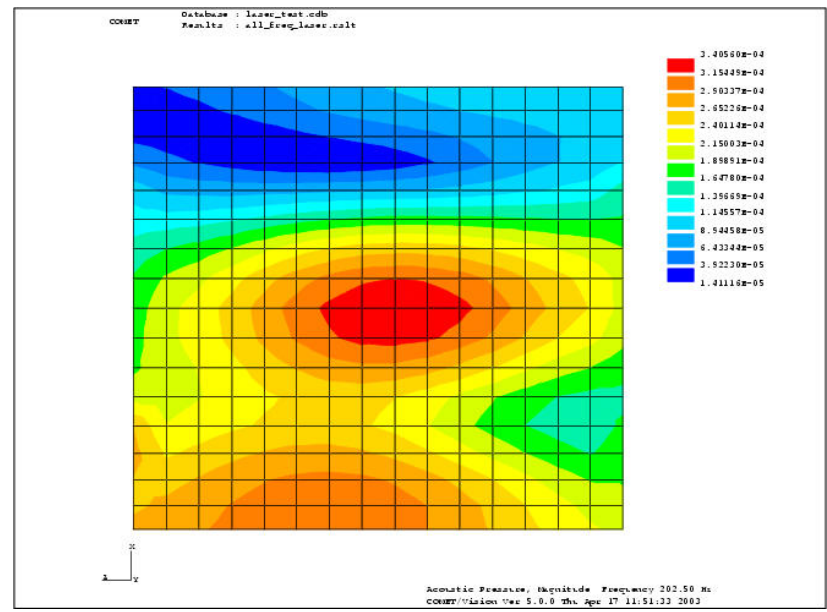

b) Magnitude of BEM predicted pressure.

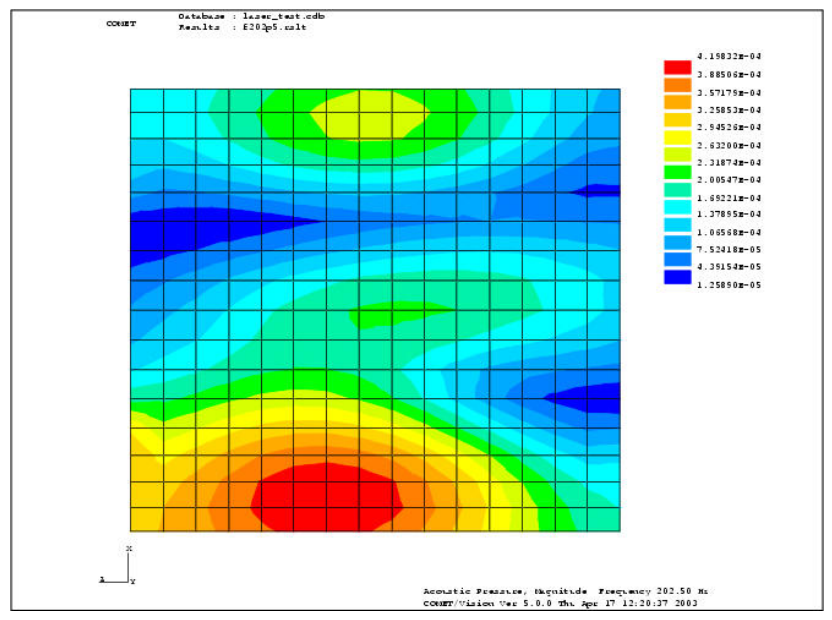

c) Magnitude of FEM-BEM predicted pressure.

Fig. 7 Comparison of spatial variation of the nearfield pressure at $202.5 \mathrm{~Hz}$ obtained from measurement, boundary element prediction using measure velocities, and finite/boundary element prediction using measured excitation, respectively.

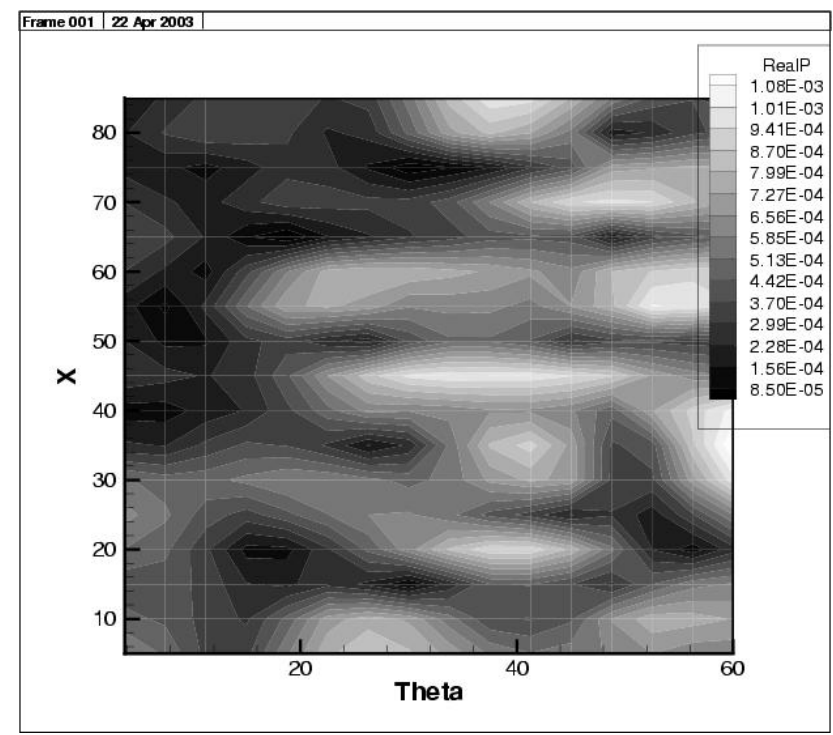

a) Magnitude of measured pressure.

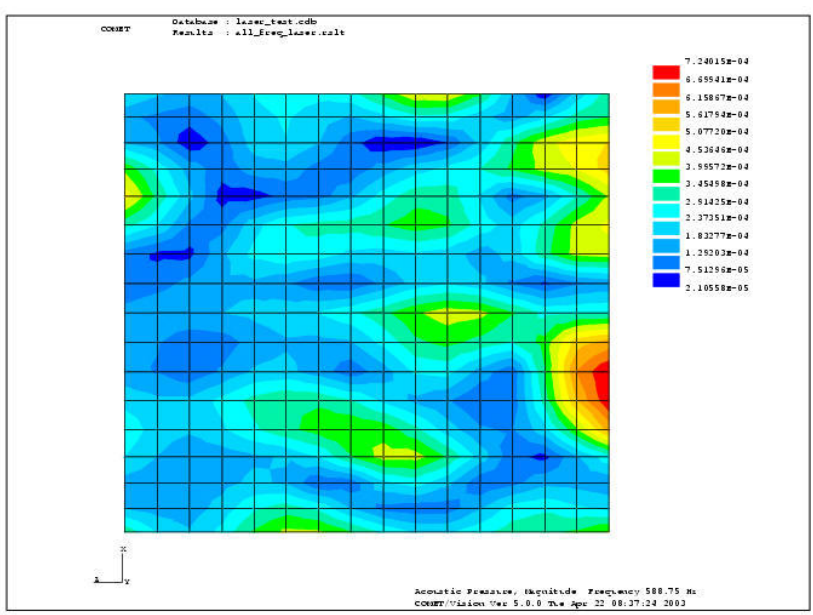

b) Magnitude of BEM predicted pressure.

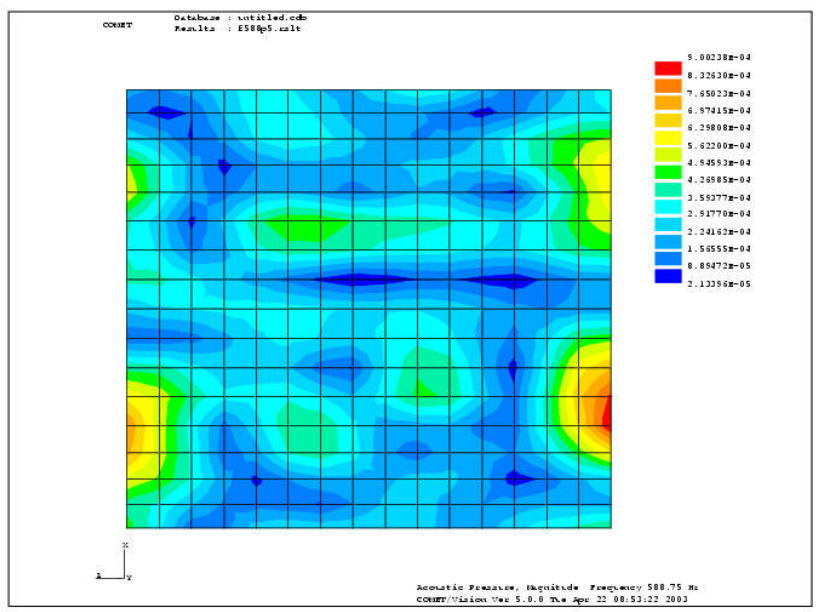

c) Magnitude of FEM-BEM predicted pressure.

Fig. 8 Comparison of spatial variation of the nearfield pressure at $588.5 \mathrm{~Hz}$ obtained from measurement, boundary element prediction using measure velocities, and finite/boundary element prediction using measured excitation, respectively. 
was designated as the equivalent mass panel. For this non physical panel the total mass of the panel was held constant as the thickness was varied. This was accomplished by artificially increasing or decreasing the density of the core material as needed to maintain a constant mass. The result of this was to provide a measures of the effect of stiffness on the radiated sound power independent of mass as a function of core thickness. The total variation in mass of the baseline panel over the range of thicknesses studied was less than 10 percent of the panels design mass.

The primary results of this parametric study are shown in Figure 9. This figure shows radiated sound power versus core thickness for the baseline and equivalent mass panels. The intersection of these two curves denotes the thickness of the panel as manufactured. It is noted that about this point the baseline panel is insensitive to changes in core thickness. It is also observed that these two curves differ over the entire range of thicknesses by what appears to be a linear function of thickness. Since the only difference between the two panels is mass, this observation seems to indicate a strong influence of mass on the sound power radiated from the panel. This observation is consistent with previously published results. To ensure that the observations are not the result of vibration modes moving out of the range of the analysis two additional plots are presented. These plots independently show the frequency content of the radiated sound power in a contour of the sound power versus frequency and thickness. Figure 10 shows this plot for the baseline configuration. The light colored streaks in this figure trace the motion of the resonant behavior of the panel as the thickness is varied. Figure 11 shows a similar plot for the equivalent mass panel. The resonant behavior of this panel is tracked by the dark colored streaks. These two figures indicate that no acoustic energy is being lost out of the low frequency range but that some energy is lost as modes move to higher frequencies out of the upper frequency range. From these plots though it is seen that this energy is negligible in terms of its effect on the overall results and particularly the sensitivity of the radiated sound power of the panel to changes in core thickness at the design point.

\section{Concluding Remarks}

The first part of this paper documents the continuing efforts to provide a validated numerical model of a curved honeycomb composite panel as installed in the SALT facility at NASA Langley Research Center for use in evaluating noise reduction concepts. Although some reasonable correlation was obtained between the measured and predicted responses the comparison is far from satisfactory. The overriding source of the problems with obtaining good correlation appears to be the choice of mounting method used to install the

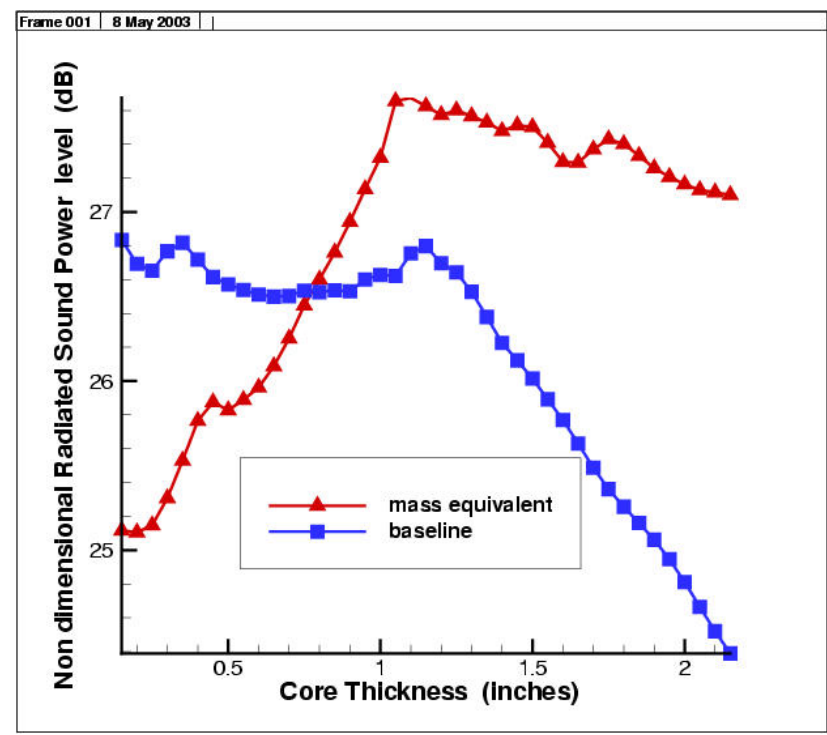

Fig. 9 Radiated sound power versus thickness for equivalent mass and constant density panels.

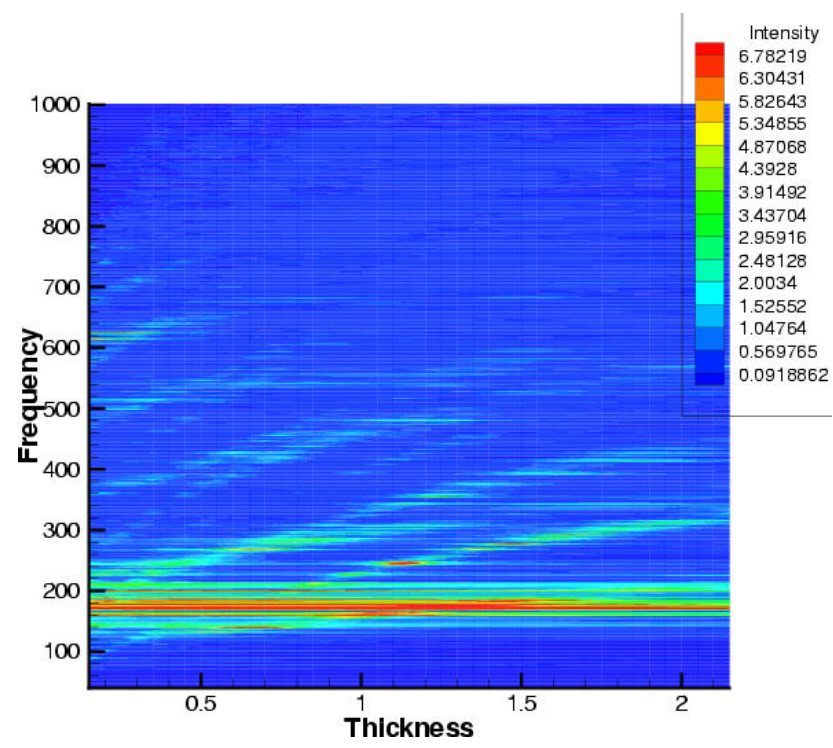

Fig. 10 Contour plot of radiated sound power versus frequency and thickness for the baseline panel.

panel in the transmission loss window. The second part of this paper presented a parametric study of the influence of core thickness on the radiated sound power from this curved honeycomb core composite panel. The basic result of this study showed that for moderate changes in core thickness about the nominal value for this panel, the radiated sound power from this panel was insensitive to core thickness. This result appeared to arise from the offsetting influences of added mass and added stiffness. The results of this study are believed to be somewhat influenced by the limited range of frequencies included in the sound power calculations but more significantly influenced by the dominance of boundary effects within this frequency range arising from the installation. 


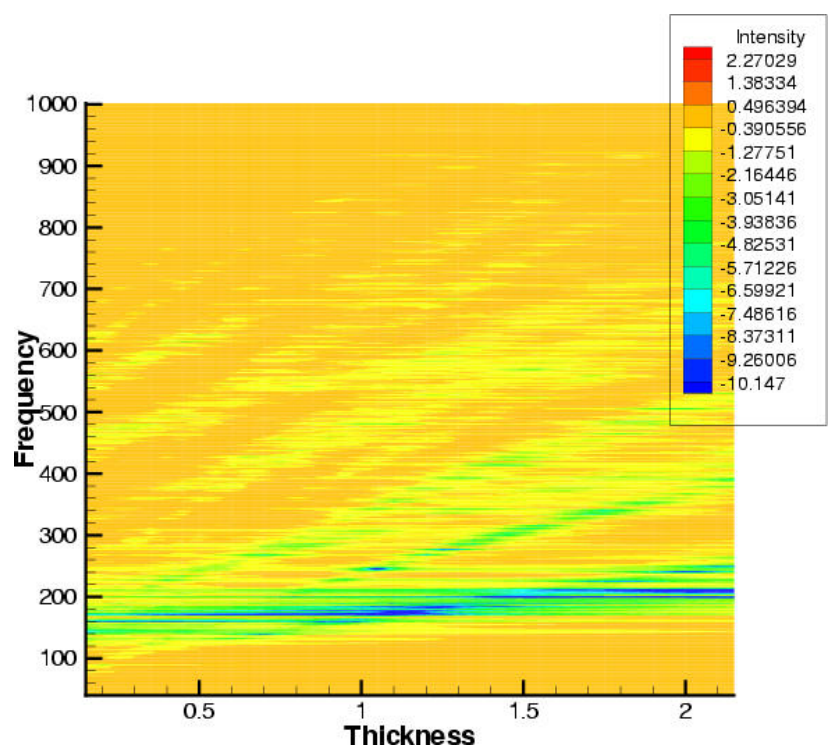

Fig. 11 Contour plot of radiated sound power versus frequency and thickness for the equivalent mass panel.

\section{References}

${ }^{1}$ Grosveld, F. W., "Calibration of the Structural Acoustic Loads and Transmission (SALT) facility at NASA Langley Research Center," Proceedings of Inter-noise 99 Fort Lauderdale, Florida, USA., 1999.

${ }^{2}$ Klos, J., Robinson, J. H., and Buehrle, R. D., "Sound Transmission Through a Curved Honeycomb Composite Panel," AIAA Conference Paper AIAA-2003-3157, May 2003.

${ }^{3}$ Buehrle, R. D., Robinson, J. H., and Grosveld, F. W., "Vibroacoustic model validation for a curved honeycomb composite panel," AIAA Conference Paper AIAA-2001-1587, Apr. 2001.

${ }^{4}$ Grosveld, F. W., Buehrle, R. D., and Robinson, J. H., "Structural and acoustic numerical modeling of a curved composite honeycomb panel," AIAA Conference Paper AIAA-20012277, May 2001.

${ }^{5}$ Leissa, A. W., "Vibration of Shells." USGPO NASA SP-288, 1973. 